nicht gefunden. - f) Oxf ph. $+29^{\circ} 22043 .-$ g) Oxf ph. ' gefunden. - n) BI - I $7^{\circ} 3933 .-$ o) BI) + i $8^{\circ} 2978 .-$ $+29^{\circ} 2073$. - h) Par ph. Klischee I $519: 362$. - i) Oxf ph. I p) (oD - $33^{\circ} 10577 .-$ q) 131$)-2^{\circ} 5186 .-$ r) 131$)+14^{\circ} 4610$. $+29^{\circ} 6506$. - j) Oxf ph. $+29^{\circ} 27179$. - k) Bord ph. Die mit $G$ bezeichneten Anschlüsse sind ron meinem Klischee $404 * 59 .-1) \mathrm{Mü}_{1} 4964 .-\mathrm{m}$ ) In Par ph. nicht, Sohn Gustav ausgefürt worden.

Genf, im Juni rq12.

F. Pidoux, Astronom.

\title{
Beobachtungen des Planeten 85 Io
}

am Kann-Refraktor (2I7 $\mathrm{mm}$ ) mit Kreuzstabmikrometer.

\begin{tabular}{|c|c|c|c|c|c|c|c|c|c|c|c|c|}
\hline \multicolumn{2}{|c|}{$\underbrace{1912}$} & M. Z. Kgst. & $A \alpha$ & $1 \delta$ & I'gl. & \multicolumn{2}{|r|}{ ce app. } & $\log p \cdot \Delta i$ & $\delta$ app. & $\log p \cdot د$ & \multicolumn{2}{|c|}{ Red.ad l. app. $\quad$ * } \\
\hline & 15 & ${ }_{1} 1^{h} 27^{m} 25^{s}$ & 90 & $-2^{\prime} 40^{\prime \prime} 1$ & I 2 & $18^{\mathrm{h}}$ & $6^{m} 17^{s} \cdot 49$ & & $2^{\prime} \quad 2.0$ & & & 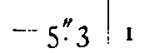 \\
\hline & 17 & I I $92 \mathbf{I}$ & $\begin{array}{lll}-1 & 10\end{array}$ & $-7 \quad 4$ & 6 & I 8 & $4 \quad 57.99$ & & $6 \quad 26.2$ & & & - \\
\hline & I 7 & $12 \quad 2422$ & -023 & -35 & IO & 18 & 45 & 9.2 & $6 \quad 36.9$ & & & \\
\hline & 23 & 12 I2 5 & to 43.40 & -03 I.2 & $\Delta$ & 18 & 120.03 & & $-3 \quad 2420.8$ & 0.840 & +2.70 & -4.9 \\
\hline
\end{tabular}

Mittlere Örter der Vergleichsterne für I9I 2.0.

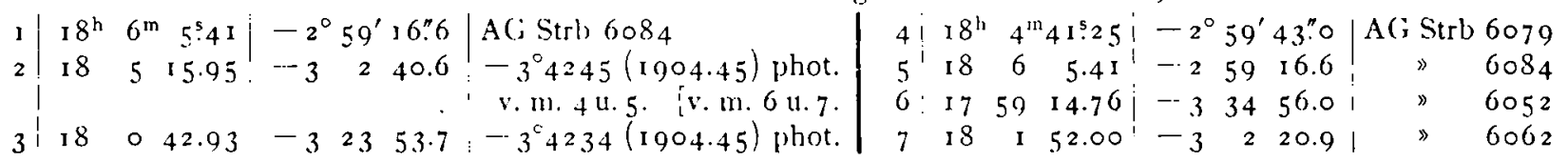

Königstuhl-Sternwarte, I 9 I 2 Aug. 15.

F. W. Panl Götz.

\section{Elements of comet 1912c (Borrelly).}

The following observations communicated by letter from the Lick Observatory were made the basis of an orbit.

I9I2 Mit. II. M. T. $\quad \alpha$ app. 3 app. Observe Nov. $4^{\mathrm{d}} 6^{\mathrm{h}} 59^{\mathrm{m}} 52^{\mathrm{s}} \times 8^{\mathrm{h}} 5^{\mathrm{m}} 58.68+35^{\mathrm{c}} 5^{\prime} 47^{\mathrm{t}} \cdot 6$ Aitken

$\begin{array}{lllllllll}7 & 13 & 16 & 18 & 53.88 & +30 & 12 & 14.9 & \end{array}$

$\begin{array}{lllllllllll} & 1 & 7 & \text { I } & 13 & \text { I } 8 & 49 & 40.96 & +24 & 7 & 3.3 .5\end{array}$

$2061350 \quad 192652.13+$ I 25.349 .7 Pitman

The elements published in I.ick Observatory Bulletin $22 \mathrm{I}$ were taken as preliminary and the first, third and fourth observations represented. 'These elements, when modified to fit the middle place exactly, gave the following residuals in the first and fourth places:

$$
\begin{array}{ccc}
O-C ; & 1 & W \\
A(x \cos \delta & -124.2 & +156.0 \\
1 \delta & +120.9 & -219.9
\end{array}
$$

These residuals were removed differentially on the assumption that a parabola would fit the observations, any outstanding residual being thrown, by the method of solution, into the declinations. The final elements referred to the mean equinox 1912 are

$$
\begin{aligned}
& T=1912 \text { Oct. } 20.8597 \text { G. M. 'T. } \\
& \omega=99^{\circ} 3 \mathbf{1}^{\prime} 53^{\prime \prime} \cdot 4 \\
& \delta 8=1434551.8 \quad 1912.0 \\
& i=12442 \text { I } 3.6 \\
& \log q=0.04385^{6} \\
& \text { Constants for the equator } 1912.0 \\
& x=\left[9.94 \cdot 5 \circ 5 \cdot r \sin \left(v+.32^{\circ} 10^{\prime} 44^{\prime \prime}\right)^{\prime}\right) \\
& y=[9.74067 \mathrm{r}] r \sin (v+1794 \mathrm{I} \quad 4.9) \\
& z=[9.984992] r \sin (v+1 \text { I } 3 \quad 37 \quad 30.7)
\end{aligned}
$$

\begin{tabular}{|c|c|c|c|c|c|c|}
\hline \multirow{2}{*}{$\begin{array}{l}1912 \\
\text { Dez. }\end{array}$} & \multirow{2}{*}{$\begin{array}{l}13 \\
25\end{array}$} & \multirow{2}{*}{$\begin{array}{c}x \text { vera } \\
6^{\mathrm{h}} 36^{\mathrm{mI}} 20^{\mathrm{s}}\end{array}$} & \multicolumn{2}{|c|}{3 vera } & \multirow{2}{*}{$\begin{array}{c}\log r \\
0.4177\end{array}$} & \multirow{2}{*}{$\begin{array}{c}\log 1 \\
0.2149\end{array}$} \\
\hline & & & $+31^{\circ}$ & 6.2 & & \\
\hline & 29 & 63144 & $+3 \mathrm{I}$ & 8.8 & & \\
\hline & 2 & $27 \quad 18$ & $+3 I$ & $9 \cdot 5$ & & \\
\hline & 6 & $\begin{array}{lll}6 & 23 & 2\end{array}$ & $+3 \mathrm{I}$ & 8.1 & 0.4240 & 0.2276 \\
\hline
\end{tabular}

These elements represent the four observations as follows:

$$
\begin{array}{ccccc}
(0-C) & \text { I } & \text { II } & \text { III } & \text { IV } \\
-(\ell \cos \delta & -0.1 & -2.5 & 0.0 & +0.3 \\
f \delta & -2.4 & - \text { I. I } & 0.0 & -1.0
\end{array}
$$

University of Kansas, I 9 I 2 I Dec. $2 . \quad$ Rejnold $K$. Young.

\section{Iva.}

Die folgende kphemeride für $\mathbf{I}^{\mathrm{h}} \mathrm{m}$. Z. Berlin beruht auf neuen Elementen, die im Anschluß an hier abgehaltene Ubungen zur Bahnbestimmung mittels 6 Beobachtungen aus ! dem Jahre 1902 berechnet sind.

$$
\begin{aligned}
& \begin{array}{ll}
\text { I9I3 } & \multicolumn{2}{c}{\alpha \text { vera }} & \text { Ev vera } \\
\text { Jan. } 106^{\mathrm{h}} \mathrm{I} 9^{\mathrm{th}} \mathrm{I}^{\mathrm{s}}+3 \mathrm{I}^{\circ} & 5.0
\end{array} \\
& 1461519+310.3 \\
& \begin{array}{lllllll}
18 & 6 \text { I } 2 & 2 & +30 & 54.2 & 0.4303 & 0.2499
\end{array} \\
& 2269 \text { io }+3047.1 \\
& 266646+3039.0 \\
& \begin{array}{lllllll}
30 & 645^{x} & +30 & 30.5 & 0.4365 & 0.2793
\end{array}
\end{aligned}
$$$$
\text { Opposition: I } 9 \mathrm{I} 2 \text { Dez. } 28 \text {. Helligkeit: }{ }^{2} 3^{\mathrm{m}} \mathrm{r}
$$

Heidelberg-Königstuhl, I 9 I 2 Dez. 20.

Inhalt zu Nr. 4622. F. A. Buchwaldt. Die Rechenlinie des Sphäroids. 253. - H. C. Plummer. On a Letter from Gauss to Argelander. 261. - F. Pidoux. Mittlere Örter einiger schwächeren, am Genfer Zehnzöller gelegentlich beobachteten und angeschlossenen Sterne. 265. - F. W. $P$. Göts. Beobachtungen des Planeten 85 10. 267 . $-K$. $K$. Young. Elements of comet $1912 \mathrm{c}$ (Borelly). 267. - A. Kopff. 497 Iva. 267.

Geschlossen $19{ }_{3}$ Jan. 6. Herausgeber 11. Kobuld. Druck von C. Sihaid. Expedition: Kicl, Multkestr. Ro. 